\section{CORRESPONDENCE \\ Combining antidepressants: understanding drug interactions is the sine qua non}

I am concerned that Palaniyappan et al's review of combining antidepressants (Palaniyappan 2009) contains imprecise genera2lisations, inaccuracies and misquotation of references.

The introduction states 'we review the nature and extent of the side-effect burden and potential risks of these combinations'. That should entail a clear exposition of pharmacodynamic and pharmacokinetic drug-drug interactions, which are the heart of the issue. Yet these two key words (and discussion of the important concepts associated with them) do not appear in Palaniyappan et al's text.

Combinations of different antidepressants are either implicitly or explicitly proscribed by various reviews and 'authorities', often the same ones who, as in this instance, get important basic facts wrong and cite references inappropriately (including mine: Gillman 2006). This is a subject about which there is already more misinformation in psychiatry texts (and in the British National Formulary) than you can shake a stick at: Palaniyappan et al's review adds to it.

I will begin by suggesting some resources that readers may access, because, without understanding the pharmacodynamics and pharmacokinetics of interactions, they will never become confident about what to do and what not to do. My own work includes a review detailing the properties, receptor potencies, cytochrome P450 (CYP450) enzyme inhibition potencies and pharmacokinetic interactions of tricyclic antidepressants (TCAs), selective serotonin reuptake inhibitors (SSRIs) and serotonin and noradrenaline reuptake inhibitors (SNRIs) (Gillman 2007); and a detailed analysis of the pharmacodynamic interaction of serotonin toxicity, explaining which MAOIs and other drugs can precipitate it (Gillman 2006). Preskorn's major review of interactions (Preskorn 2006) is highly recommended, as are his website (www.preskorn. com) and that of Flockhart (http://medicine.iupui. edu/clinpharm/ddis) and the Allele Nomenclature Committee (www.cypalleles.ki.se). Most other web sources and software programs are inaccurate. Other key papers on CYP450 enzymes are: Brosen 2004; Ingelman-Sundberg 2005a,b; Sim 2006.

It is also notable that there are great differences between tranylcypromine (TCP) and phenelzine (PLZ), especially with regard to CYP450 interactions (Holt 2004): briefly, PLZ is a mechanismbased (i.e. irreversible) inhibitor of most CYP450 enzymes, whereas TCP is not, except for nanomolar (reversible) potency for CYP2A6 inhibition
(Draper 1997). These differences affect potential interactions.

Palaniyappan et al cite my review of the TCAs (Gillman 2007) but seem to disregard most of its content and produce an unreferenced interaction table out of step with that (and other) sources. They state, concerning my comments on dual action strategies: 'The combination of a predominantly noradrenergic TCA such as nortriptyline and an SSRI may overcome this ceiling effect and produce a different sodium:5-HT reuptake blockade ratio. However, there is no evidence that this ratio is related in any way to clinical effectiveness.'

Aside from the rendering of 'NA:5-HT' as 'sodium:5-HT', the quote indicates a misunderstanding of what I discussed, which had nothing to do with ceiling effects. Quite the opposite in fact: I argued that most SNRIs, but particularly venlafaxine, exhibit a subtherapeutic noradrenergic effect, as evidenced by low affinity at the human cloned noradrenaline transporter, and no substantial effect on the tyramine pressor response (Blier 2007; Debonnel 2007) - a ‘floor' effect perhaps? Therefore, if it were possible to attain full noradrenaline reuptake inhibition, the dose required would be about 10 times the maximum dose of $300 \mathrm{mg}$, i.e. a toxic level (Whyte 2003). In fact, this extremely weak NRI is the rationale for adding reboxetine, to attain a more balanced SNRI effect. So their comment (regarding venlafaxine + reboxetine) is wrong: 'Any synergism of such a combination is doubtful, as both drugs act via the same mechanism; the same effects could be achieved by a higher dose of venlafaxine alone, with more predictable pharmacokinetics' - indeed, predictably toxic.

Also, their claim that 'there is no evidence that this ratio is related in any way to clinical effectiveness' denies the established reality, logic and basic pharmacology of the dose-response curve. Since venlafaxine has approximately a 200:1 differential between 5-HT:NA transporter affinity it is impossible to have an optimum therapeutic: side-effect ratio for both systems simultaneously, or even any meaningful balanced effect on both systems simultaneously. The tacit acceptance by psychiatrists of the notion that venlafaxine has an SNRI effect is astonishing, because it is closer to myth than reality. Contrary to Palaniyappan et al's assertion, there is both theoretical and clinical evidence that dual action (a true SNRI effect) is more effective. Nelson et al's randomised controlled trial (RCT) of fluoxetine and desipramine indicated much higher remission rates at 6 weeks for combined treatment (54\%) than for the SSRI (7\%) or desipramine (0\%) alone (Nelson 2004). Palaniyappan et al also misrepresent that trial by reporting: 'In any event, this speed of onset effect could not be replicated in 
a later RCT (Nelson 2004)', without mentioning the remission rate. They also use this same reference a second time, implying that it is subsequent work.

Their material and references on my area of expertise, serotonin toxicity, are poor for a review article. The section headed 'SSRI with moclobemide' shows a lack of familiarity with the literature on reversible inhibitors of monoamine oxidase A (RIMAs) and SSRIs (Gillman 2006). One of several examples of this is: 'The SSRImoclobemide combination has been tried with the same rationale as the SSRI-MAOI combination. Three small open-label trials (total $n=46$ ) found moclobemide to be effective in combination with SSRIs (Dodd 2005).'

Dodd, their most frequently cited reference, is another review. It is not the original source of any data. There is limited profit in one review (mis)quoting another review. Palaniyappan et al misrepresent the RIMA/SSRI area of research. They misreport Dodd (who cites two open-labelled studies (Joffe 1994; Hawley 1996), not three). Hawley's original report (Hawley 1996) had 50 cases (cited by Dodd, correctly), taking the total beyond their stated 46 (Table 2). They might have noted that Hawley stated, concerning adverse drug reactions, 'Many events were rated as severe. The high rate of adverse events suggests that there are clinically significant pharmacodynamic interactions between moclobemide and SSRIs' (i.e. serotonin toxicity). Note that there are two other moclobemide/SSRI papers (Bakish 1995; Ebert 1995), although Ebert was not cited by Dodd.

Why, one might ask, despite the great enthusiasm expressed by all of these authors, has nothing else been published in the subsequent decade? I sought information on this point, repeatedly, from all those authors, but only Dr Hawley ever replied (see below).

My detailed analysis of data on animal and human serotonin toxicity, and of that regarding moclobemide in particular (Gillman 2004, 2006), provides a more substantive basis for understanding the risks of combining moclobemide and SSRIs. Among other things, my review states that 'Hawley decided to stop his research because of high levels of moderately severe serotonergic side effects', i.e. incipient serotonin toxicity. Readers of Palaniyappan et al's article are likely to be left with the impression that SSRI/moclobemide combinations are'relatively safe'. That notion is seriously contentious and the contrary evidence should have been referenced and discussed. I am supposed to be an expert in serotonin toxicity and I would not be game to try it. The answer to the third MCQ question is wrong: moclobemide + SSRI definitely could cause fatal serotonin toxicity.
The format of Table 1 is unsuitable for conveying that type of data and will probably mislead and confuse many readers. It, and the associated text, has some odd material that does not promote a good understanding of pharmacology, interactions, or CYP450 enzymes, and it is less useful than other pre-existing sources and references given here. It is, inexcusably, unreferenced. The authors state (under the heading, and in the context of, combinations of an SSRI with a TCA) 'Tricyclic toxicity ... is a particular risk for the $7 \%$ of White people who lack sufficient CYP2D6 to metabolise TCAs (Albers 1996)'. This appears to be muddled thinking because that is precisely the group in whom an interaction is less of a risk, because they are already genotypic poor metabolisers and further diminution of CYP450 activity (by SSRIs) will not make them worse; such people are at increased risk of toxicity with monotherapy with TCAs. Albers is an outdated and inappropriate reference. In my opinion readers would do better to consult the following more helpful and accurate sources: Brosen 2004; Ingelman-Sundberg 2005a; Preskorn 2006; Sim 2006; Gillman 2007; Flockhart 2009; Preskorn's extensive review contains a wealth of good information.

There are various other errors in the article, which is characterised by imprecision, unhelpful generalisations and uncritical repetition of other reviews. In my opinion anyone contemplating using combinations needs to be rather better informed than they would become by reading Palaniyappan et al.

Albers LJ, Reist C, Helmeste D, et al (1996) Paroxetine shifts imipramine metabolism. Psychiatry Research; 59: 189-96.

Bakish D, Hooper CL, West DL, et al (1995) Moclobemide and specific serotonin reuptake inhibitor combination treatment of resistant anxiety and depressive disorders. Human Psychopharmacology; 10: 105-9.

Blier P, Saint-Andre E, Hebert C, et al (2007) Effects of different doses of venlafaxine on serotonin and norepinephrine reuptake in healthy volunteers. International Journal of Neuropsychopharmacology; 10 : 41-50.

Brosen K (2004) Some aspects of genetic polymorphism in the biotransformation of antidepressants. Therapie; 59: 5-12

Debonnel G, Saint-André É, Hébert C, et al (2007) Differential physiological effects of a low dose and high doses of venlafaxine in major depression. International Journal of Neuropsychopharmacology; 10: $51-61$

Dodd S, Horgan D, Malhi GS, et al (2005) To combine or not to combine? A literature review of antidepressant combination therapy. Journal of Affective Disorders; 89: 1-11.

Draper AJ, Madan A, Parkinson A (1997) Inhibition of coumarin 7-hydroxylase activity in human liver microsomes. Archives of Biochemistry and Biophysics; 341: 47-61.

Ebert D, Albert R, May A, et al (1995) Combined SSRI-RIMA treatment in refractory depression. Safety data and efficacy. Psychopharmacology; 119: 342-4.

Flockhart DA (2009) Drug Interactions: Cytochrome P450 Drug Interaction Table. Indiana University School of Medicine (http://medicine.iupui.edu/ clinpharm/ddis/table.asp). 
Gillman PK (2004) Moclobemide and the risk of serotonin toxicity (or serotonin syndrome). Central Nervous System Drug Reviews; 10: 83-5.

Gillman PK (2006) A review of serotonin toxicity data: implications for the mechanisms of antidepressant drug action. Biological Psychiatry: 59: 1046-51.

Gillman PK (2007) Tricyclic antidepressant pharmacology and therapeutic drug interactions updated. British Journal of Pharmacology; 151: 737-48 [Free full text].

Hawley CJ, Quick SJ, Ratnam S, et al (1996) Safety and tolerability of combined treatment with moclobemide and SSRIs - a systematic study of 50 patients. International Clinical Psychopharmacology; 11: 187-91.

Holt A, Berry MD, Boulton AA (2004) On the binding of monoamine oxidase inhibitors to some sites distinct from the MAO active site, and effects thereby elicited. Neurotoxicology; 25: 251-66.

Ingelman-Sundberg M (2005a) Genetic polymorphisms of cytochrome P450 2D6 (CYP2D6): clinical consequences, evolutionary aspects and functional diversity. Pharmacogenomics Journal; 5: 6-13 [Free full text].

Ingelman-Sundberg M, Rodriguez-Antona C (2005b) Pharmacogenetics of drug-metabolizing enzymes: implications for a safer and more effective drug therapy. Philosophical Transactions of the Royal Society of London. Series B: Biological Sciences; 360: 1563-70.

Joffe RT, Bakish D (1994) Combined SSRI-moclobemide treatment of psychiatric illness. Journal of Clinical Psychiatry; 55: 24-5.

Nelson JC, Mazure CM, Jatlow PI, et al (2004) Combining norepinephrine and serotonin reuptake inhibition mechanisms for treatment of depression: a double-blind, randomized study. Biological Psychiatry; 55: 296-300.

Palaniyappan L, Insole L, Ferrier N (2009) Combining antidepressants: a review of evidence. Advances in Psychiatric Treatment, 15: 90-9.

Preskorn S, Flockhart D (2006) 2006 Guide to psychiatric drug interactions. Primary Psychiatry; 13: 35-64.

Sim SC, Ingelman-Sundberg M (2006) The human cytochrome P450 Allele Nomenclature Committee Web site: submission criteria, procedures, and objectives. Methods in Molecular Biology; 320: 183-91.

Whyte IM, Dawson AH, Buckley NA (2003) Relative toxicity of venlafaxine and selective serotonin reuptake inhibitors in overdose compared to tricyclic antidepressants. Quarterly Journal of Medicine; 96: 369-74 [Free full text].

Ken Gillman MD, MRCPsych, PsychoTropical Research,

P0 Box 86 Bucasia 4750, Mackay, Queensland, Australia.

Email: kg@matilda.net.au

doi: 10.1192/apt.16.1.76

\section{Authors' reply}

Some of Dr Gillman's trenchant criticisms arise from an apparent misunderstanding over the type of article we have written. Therefore we thought it helpful to give some background to the nature of the article before responding to the individual critiques.

The remit of the article was to review the efficacy and side-effect burden of antidepressant combinations reported in the clinical literature. Therefore, exploring specific pharmacokinetic aspects of each combination is outside the scope of this work, although we have highlighted important pharmacodynamic rationales for the combinations wherever possible. We welcome the addition of more references from Dr Gillman but we must emphasise that our original article was constrained by the limits of the journal style. Advances in Psychiatric Treatment is an aid for CPD that publishes reviews rather than detailed data papers and requires only a limited reference list that is accessible to readers. In many instances we therefore used secondary references that discuss the primary data papers. As indicated in the article, a fuller list of references is available on request. Table 1 contains no references but the data in it are taken from references listed throughout the review.

In keeping with the objectives of this journal a section of self-assessment follows every article. This self-assessment exercise should be in line with the Royal College of Psychiatrist's Membership Examination as closely as possible. The MCQs that follow our article are in the 'best of five' format. The reader chooses the best of five responses and this does not mean that the other responses are necessarily wrong.

Turning to the specifics, we first of all apologise for the error in copy-editing rightly pointed out by Dr Gillman. The text discussing SSRIs and TCA combinations should read 'NA:5HT reuptake blockade' and not 'sodium:5HT reuptake blockade'. We also stand corrected with the numbers reported in the SSRI/RIMA section. It should read 'Two small open-label trials (total $n=61$ )'.

The effectiveness of a drug in randomised controlled trials (RCTs) is a different domain from assessing the pharmacodynamics and pharmacokinetics of compounds in the laboratory. We wish to underline the weaknesses of Nelson's RCT evaluating the desipramine and fluoxetine combination (Nelson 2004). First, the sample size was very small (39 participants, 1 of whom dropped out and another was excluded) and second, the baseline Montgomery-Åsberg Depression Rating Scale (MADRS) scores were lower in the combined treatment group (which nearly reached significance at $P=0.07$ ). This trial did not show a significant difference between the groups when the endpoint MADRS scores were compared. Although the mean percentage change in MADRS was numerically higher in the combined treatment group, this again failed to reach statistical significance. When categorical levels of treatment response were considered, the percentages of remitters in this 6 -week follow-up trial were $54 \%$ for the combined treatment, $7 \%$ for fluoxetine and $0 \%$ for desipramine. However, when all responders (total achieving categorical remission + categorical response) are considered, the combined treatment was only marginally better ( 8 out of 13 in the combined group v. 6 out of 14 in the fluoxetine group). The percentage of 'non-responders' in the 